\title{
Interactive health insight miner: an adaptive, semantic-based approach
}

\author{
Isabel Funke \\ isabel.funke@gmail.com
}

\author{
Rim Helaoui and Aki Härmä \\ Philips Research \\ High Tech Campus 34 \\ 5656 AE Eindhoven \\ The Netherlands \\ rim.helaouidphilips.com \\ aki.harma@philips.com
}

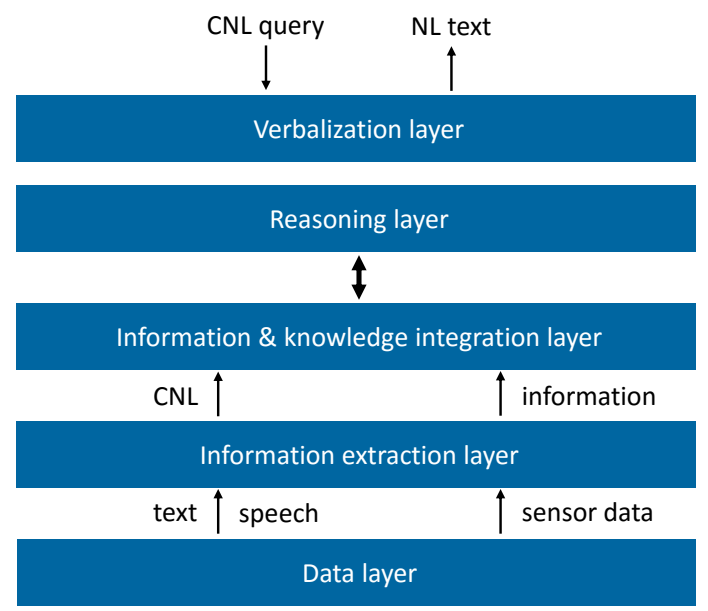

Figure 1: Proposed framework for the interactive health insight miner.

the extracted information into formal facts. The resulting knowledge base can include user- and situation-specific information as well as common sense knowledge. The reasoning layer leverages logic-based algorithms that reason with the available knowledge. The verbalization layer transforms the facts into coherent and comprehensible natural language (NL) messages. Similar systems for data-to-text summarization have been proposed in the literature (e.g. Portet et al., 2009).

We additionally introduce a Controlled Natural Language ( $C N L)$. It is a formal language that can be translated unambiguously into knowledge base facts, but is also understandable by humans. By adopting the CNL, the user can interact with the system, i.e., add and query facts from the knowledge base. Natural language or spoken text can be fed into the system after translation into CNL.

\section{Representing, summarizing and verbalizing insights}

The user's lifestyle is described by an ontology that contains the routines, habits, and targets of the 
user. These concepts are leveraged to represent insights as knowledge base facts.

Inspired by NaturalOWL (Galanis and Androutsopoulos, 2007), we include lexical annotations in the ontology, which specify how ontology concepts are to be translated into natural text. This way, the ontology also acts as a lexicon. We include the lexical categories (e.g., noun, determiner, preposition, or verb) in the annotations to facilitate the use of standard Natural Language Generation (NLG) techniques, such as adapting verb conjugations, adapting the verb tense, or aggregating sentence parts.

To enable user interaction, we specify a CNL based on the vocabulary defined in the ontology. The CNL plays the role of a human and machine understandable interface which allows to directly map the user's input to the formal concepts of the ontology. This way, the user can add personal information to the system, e.g., "On Monday at work, I play tennis". This statement will be formalized as a fact and added to the knowledge base. The CNL also provides the basis for verbalizing the system's responses to the user's queries, such as "What are insights about Sunday afternoon?" We use the Backus-Naur form to specify the CNL as a context-free grammar.

To create an NL summary of a number of insights, we implement the following NLG steps (Bouayad-Agha et al., 2014): (1) Content selection: We let the user ask for specific insights, for example insights about their step count on Sunday. (2) Discourse planning: We group those insights together that are semantically related. The insights are first grouped by the measurement to which they refer (e.g. step count) and then ordered within each group from more general to more specific. For the grouping and ordering steps, we leverage our semantic model (ontology) and apply reasoning algorithms to determine which relationships hold between which insights. (3) Lexicalization: We follow a template-based approach using the lexical annotations in the ontology. (4) Aggregation: We verbalize each group of equally specific insights using an aggregation template. Finally, for (5) realization, we use the realization engine SimpleNLG (Gatt and Reiter, 2009).

\section{Implementation}

We demonstrate the viability of our framework by implementing one use case related to lifestyle insight mining. The ontology is implemented in OWL using the Protégé ${ }^{1}$ editor. We use OWL $\mathrm{API}^{2}$ 4.1.3 together with the reasoner Her$\mathrm{miT}^{3}$ 1.3.8 to implement our system in Java. To implement the verbalization functionality, we build upon the library SimpleNLG ${ }^{4}$.

\section{Example run}

$>$ What are insights about my sedentary time on Sunday?

$>$ On Sunday your sedentary time is lower than on Tuesday, Friday, Wednesday or Thursday.

On Sunday in the afternoon your sedentary time is lower than on Friday, Wednesday, Thursday or Tuesday in the afternoon.

$>$ What are insights about when I go running?

$>$ Could not find any insights about this.

$>$ On Sunday in the afternoon, I go running.

$>$ What are insights about when I go running?

$>$ On Sunday in the afternoon when you go running your sedentary time is lower than on Tuesday, Friday, Thursday or Wednesday in the afternoon.

\section{References}

Nadjet Bouayad-Agha, Gerard Casamayor, and Leo Wanner. 2014. Natural language generation in the context of the semantic web. Semantic Web, 5(6):493-513.

Dimitrios Galanis and Ion Androutsopoulos. 2007. Generating multilingual descriptions from linguistically annotated OWL ontologies: the NaturalOWL system. In Proceedings of the 11th European Workshop on Natural Language Generation, pages 143146.

Albert Gatt and Ehud Reiter. 2009. SimpleNLG: A realisation engine for practical applications. In Proceedings of the 12th European Workshop on Natural Language Generation, pages 90-93.

Aki Härmä and Rim Helaoui. 2016. Probabilistic scoring of validated insights for personal health services. In 2016 IEEE Symposium Series on Computational Intelligence (SSCI), pages 1-6.

François Portet, Ehud Reiter, Albert Gatt, Jim Hunter, Somayajulu Sripada, Yvonne Freer, and Cindy Sykes. 2009. Automatic generation of textual summaries from neonatal intensive care data. Artificial Intelligence, 173(7-8):789-816.

\footnotetext{
${ }^{1}$ http://protege.stanford.edu/

${ }^{2}$ https://github.com/owlcs/owlapi

${ }^{3}$ http: / / www . hermit-reasoner.com/

${ }^{4}$ https://github.com/simplenlg/ simplenlg
} 